\title{
ANALYSIS OF ESCHERICHIA COLI O104:H4 OUTBREAK IN GERMANY IN 2011 USING DIFFERENTIATION METHOD FOR UNUSUAL EPIDEMIOLOGICAL EVENTS
}

\author{
Vladan Radosavljević ${ }^{1,2}$, Ernst Jürgen Finke ${ }^{3}$, Goran Belojević ${ }^{4}$ \\ ${ }^{1}$ Military Academy, University of Defense, Belgrade, Serbia \\ ${ }^{2}$ Medical Corps Headquarters, Army of Serbia, Belgrade, Serbia \\ ${ }^{3}$ Specialist in Microbiology, Virology and Infection Epidemiology, Munich, Germany \\ ${ }^{4}$ Institute of Hygiene and Medical Ecology, Faculty of Medicine, University of Belgrade, Belgrade, Serbia
}

\begin{abstract}
SUMMARY
Aim: The aim of the study was to further clarify the origin of Escherichia coli 0104:H4 outbreak in Germany in 2011 (German Ec) as the likelihood of a deliberate act has not been excluded in previous analyses.

Methods: We use an original and the most detailed scoring method so far, with 33 parameters pertaining to the source of infection/reservoir or possible perpetrator, pathogen or biological agent, transmission mechanism/factors or means/media of delivery, and population at risk or target.

Results: Total scores for a deliberate or accidental epidemic indicate that the outbreak was more probably caused unintentionally, presumably due to technical accidents or hygienic shortcomings in the food chain.

Conclusions: The validity of the present assessment is limited by the lack of data on the reservoir of the pathogen, the source of infection, and the mode of food contamination. Conclusive evidences on these parameters are essential for the final clarification of the outbreak origin.
\end{abstract}

Key words: Escherichia coli, Shiga toxin, haemolytic-uremic syndrome, gastroenteritis, epidemics, Germany

Address for correspondence: G. Belojević, Institute of Hygiene and Medical Ecology, Faculty of Medicine, University of Belgrade, Dr Subotica 8, 11000 Belgrade, Serbia. E-mail: goran.belojevic@hotmail.com

http://dx.doi.org/10.21101/cejph.a4255

\section{INTRODUCTION}

From 1 May to 5 July 2011 Germany faced the largest and the most severe food-borne outbreak of haemolytic- uremic syndrome (HUS) ever. It was reported to the World Health Organization (WHO) according to the International Health Regulations (1). The outbreak was caused by the unique serotype O104:H4 of Shiga toxin producing Escherichia coli, termed also as enterohaemorrhagic Escherichia coli (EHEC) (1). There were 2,987 cases of bloody diarrhoea (18 lethal) and 855 cases complicated with HUS (35 lethal). Additionally, 83 German Ec cases and 54 patients with HUS were recorded outside Germany, most of them linked to travelling to Northern Germany in May 2011 (2). German authorities and the European Food Safety Authority (EFSA) announced that the contaminated sprouts grown from fenugreek seeds imported from Egypt in 2009 were the most probable vehicle of the pathogen in this epidemic (1).

Based on the results of a comparative study performed with three models for differentiation between natural and deliberate epidemics (3), the German Ec outbreak may be regarded as an unusual epidemiological event (UEE). This assumption is supported by the following epidemiological, demographical, biological, microbiological, pathological, and clinical features of German Hemolytic Uremic Syndrome/Enterohemorrhagic Escherichia
Coli (GHUSEC): high pathogenicity and extended spectrum beta-lactamase antibiotic resistance of a chimeric (enterohaemorrhagic/enteroaggregative) O104:H4 strain (1, 4-10), sudden appearance and disappearance of the pathogen (1), suitability for genetic manipulation (11), long term carriage and excretion of the pathogen $(9,11,12)$, easy pathogen spreading by different transmission routes $(9,12)$, longer incubation period than typical for known EHEC serotypes $(1,12)$, and no definite conclusions about the use of antibiotics in acute EHEC diarrhoea as well as for the causal therapy at all (2). There was a rapidly increasing number of patients with severe diarrhoea and unusually high proportion of patients with HUS, very fast supra-regional spread throughout Germany and in 13 other European countries plus cases in the USA and Canada and unexpectedly high incidence in adults and females (13-15). The causative role of fenugreek seeds exported from Egypt was questionable (12) due to difficulties to isolate the pathogen from fenugreek seeds and to identify sources of infection, reservoirs and modus of contamination of the food (1). The outbreak was unusually long-lasting, especially considering peaceful time without natural disasters in a highly developed country with a prominent healthcare system.

One of the key points of the recent comparative study of the unique German Ec outbreak (3) was that the pathogen could have been introduced in the food chain deliberately. In order to further 
assess the probable origin of this epidemic we use a more detailed and subtle retrospective assessment.

\section{MATERIALS AND METHODS}

A literature review was performed in the database MEDLINE for the period 1999-2014 in order to obtain necessary data for a retrospective analysis of the German Ec outbreak with the following key words: outbreak, epidemic, Escherichia coli, EHEC O104:H4, STEC O104:H4, HUS, gastroenteritis, haemolytic uremic syndrome, HUS, sprouts, seeds, and Germany.

We analysed the German Ec outbreak using a subtle and detailed differentiation method for four possible UEE scenarios: natural outbreak of a known endemic disease; natural outbreak of a new or re-emerging disease; outbreak by an accidental release of a pathogen; outbreak by a deliberate delivery of a biological agent. This method is based on 23 qualitative and 10 quantitative parameters (16-17). Parameters with its characterizing features were grouped in four components of the chain of infection (Table 1): Reservoir/source of infection vs. perpetrator: sophistication, motivation, intention, intelligence, secrecy, number of perpetrators, number of reservoirs/sources of infection, accessibility to sources of pathogens or biological agents, accessibility to susceptible population or targets; Pathogen vs. biological agent: A category, $\mathrm{B}$ category, $\mathrm{C}$ category, emerging pathogen, amount of the pathogen or available agent; Factors of transmission vs. means/media of delivery: air, water, food, fomites, vectors (e.g. arthropods), ammunition, delivery systems/devices and dispersion systems/devices, mechanisms of release; Susceptible population vs. target: intelligence, secrecy, personal control, control of means/ media, physical protection, protection by chemoprophylaxis, protection by immuno-prophylaxis, importance of a target ("soft" or "hard" target), number of people at risk/in a target, location of people at risk/in a target, distribution of people at risk/in a target.

Each parameter was scored with 1 or 0 , depending on the assessed high or low probability, respectively. If there were no data to assess a parameter the term N/A (not applicable) was used. Total scores of $0-8,9-16,17-24$, and $25-33$ indicate lowly probable, possible, highly probable, and certain type of outbreak, respectively (Table 1).

The differentiation approach includes the system of elimination. If all parameters of at least one of four components are scored with 0 or N/A the appropriate scenario is not considered as possible (17).

\section{RESULTS}

The specific parameters and features of the differentiation method and the scores for possible epidemic scenarios are presented in Table 1.

\section{Natural Outbreak Scenarios}

No acute illnesses among humans or animals by the implicated EHEC O104:H4 strain as natural source of infection were identified in or outside Germany during and after UEE (1). Furthermore, there had been no natural disasters with damages of the ecologi- cal system, infrastructure or hygienic barriers that could cause a massive contamination of the food chain and development of this explosive epidemic. These arguments suggest that the GHUSEC event was not a natural outbreak of an endemic disease (NE).

A natural outbreak of a new or re-emerging disease (NR) can also be excluded despite the fact that the pathogen differs in some biological and genetic characteristics from the known EHEC serotypes. Acute gastroenteritis with or without bloody diarrhoea and/or enteropathic HUS caused by EHEC are already endemic in Germany and have to be notified (18).

In the case of NE and NR five qualitative and one quantitative parameter for the assessment of the component perpetrator/ source of infection/reservoir were not applicable. Natural source/ reservoir of infection was not found in Germany, and it is not possible to repeat such outbreak in the same natural conditions. Therefore, the last three parameters from this component are scored with zero. According to the system of elimination NE and NR scenarios were excluded from further analysis, because one component contains all parameters scored with 0 or N/A (17).

High intensity and dynamic of the initial epidemic phase, with sudden occurrence of multiple clusters of cases within the maximum incubation period of the pathogen after having meals mainly in restaurants or cafeterias was more likely characteristic for an outbreak caused by accidental release of a pathogen (AR) or a deliberate outbreak (DO) (19).

In the absence of natural sources of infection, the contaminated sprouts were considered as an equivalent for the origin, or a causative source of the explosive primary wave of epidemic in order to assess the parameters.

\section{Accidental Release and Deliberate Outbreak Sce- narios}

\section{Perpetrator}

There is a likelihood that modern food chains might be harmed by individuals or groups with the appropriate scientific-technical sophistication, criminal or political motivation and intentions and accessibility to sources of agents and sensitive targets of interest (each parameter scored with "one" for deliberate outbreak). Intelligence e.g. the ability to get direct, unambiguous, objective and timely information on global and local levels related to a biological attack is provided by different German federal ministries, but in this UEE no appropriate evidence was notified by them. Secrecy comprises the capacity to keep activities unobserved before a deliberate outbreak starts and to hold perpetrators unknown during and after an outbreak (16). Besides a general perception of biological risks we have no reliable data on the number of perpetrators or suspect activities before or coincident with the German Ec outbreak (score 0 for these three conclusive parameters).

\section{Source (Vehicle) of Infection}

If an accidental release is assumed the deliberate outbreak specific parameters "sophistication, intention, motivation" and "number of perpetrators" are not applicable. As the outbreak was managed very openly by media, national and international public health and consumer protection authorities we scored "intelligence" with 1 and "secrecy" with 0 . 
Table 1. Assessment of the Escherichia coli 0104:H4 outbreak in Germany in 2011 by differentiation scoring for a natural outbreak of an endemic disease (NE), a natural outbreak of a new or re-emerging disease (NR), an outbreak by an aaccidental release of a pathogen $(A R)$ and a deliberate outbreak (DO)

\begin{tabular}{|c|c|c|c|c|}
\hline Parameter & $\mathrm{NE}$ & NR & AR & DO \\
\hline \multicolumn{5}{|l|}{ Perpetrator/source of infection/reservoir of pathogen } \\
\hline Sophistication & $\mathrm{N} / \mathrm{A}$ & $\mathrm{N} / \mathrm{A}$ & $\mathrm{N} / \mathrm{A}$ & 1 \\
\hline Motivation & $\mathrm{N} / \mathrm{A}$ & $\mathrm{N} / \mathrm{A}$ & $\mathrm{N} / \mathrm{A}$ & 1 \\
\hline Intention & $\mathrm{N} / \mathrm{A}$ & $\mathrm{N} / \mathrm{A}$ & $\mathrm{N} / \mathrm{A}$ & 1 \\
\hline Intelligence & $\mathrm{N} / \mathrm{A}$ & $\mathrm{N} / \mathrm{A}$ & 1 & 0 \\
\hline Secrecy & $\mathrm{N} / \mathrm{A}$ & $\mathrm{N} / \mathrm{A}$ & 0 & 0 \\
\hline Number of perpetrators & $\mathrm{N} / \mathrm{A}$ & N/A & $\mathrm{N} / \mathrm{A}$ & 0 \\
\hline Number of sources of infection/reservoirs & 0 & 0 & 1 & 1 \\
\hline Accessibility to sources of agent/pathogen & 0 & 0 & 1 & 1 \\
\hline Accessibility to targets/population at risk & 0 & 0 & 1 & 1 \\
\hline \multicolumn{5}{|l|}{ Biological agent/pathogen } \\
\hline A category* & & & 0 & 0 \\
\hline B category* & & & 1 & 1 \\
\hline C category* & & & 0 & 0 \\
\hline Emerging pathogen & & & 1 & 1 \\
\hline Amount of the available agent/pathogen & & & 1 & 0 \\
\hline \multicolumn{5}{|l|}{ Means/media of delivery/factors of transmission } \\
\hline Air & & & 0 & 0 \\
\hline Food & & & 1 & 1 \\
\hline Water & & & 0 & 0 \\
\hline Fomites & & & 0 & 0 \\
\hline Vectors & & & 0 & 0 \\
\hline Biological ammunition & & & $\mathrm{N} / \mathrm{A}$ & 0 \\
\hline Delivery systems & & & 1 & 0 \\
\hline Dispersion systems/mechanism of release & & & 1 & 0 \\
\hline \multicolumn{5}{|l|}{ Target/susceptible population at risk } \\
\hline Intelligence & & & 1 & 0 \\
\hline Secrecy & & & 0 & 0 \\
\hline Personal control & & & 1 & 0 \\
\hline Control of means/media of delivery/factors of transmission & & & 1 & 0 \\
\hline Physical protection & & & 0 & 0 \\
\hline Protection by chemoprophylaxis & & & 0 & 0 \\
\hline Protection by immuno-prophylaxis & & & 0 & 0 \\
\hline Importance of target/population at risk & & & 1 & 1 \\
\hline Location of target/population at risk & & & 1 & 0 \\
\hline Number of people in a target/population at risk & & & 1 & 0 \\
\hline Distribution of people in a target/population at risk & & & 1 & 0 \\
\hline Total & - & - & 17 & 10 \\
\hline
\end{tabular}

${ }^{*}$ CDC classification

$0=$ low probability, 1 = high probability, N/A = not applicable/no data, $-=$ eliminated from further consideration

Total Scores: $0-8=$ lowly probable type of outbreak (TO); $9-16=$ possible TO; $17-24=$ highly probable TO; $25-33=$ certain TO.

This explosive food-borne outbreak could develop only due to the accessibility to the pathogen by a probably accidental contamination of fenugreek sprouts and extensive exposure to presumably high number of this vehicle after its federal wide distribution. The latter provided the pathogen's efficient accessibility to susceptible populations at risk via the food chain. These three quantitative parameters were scored with 1 for the accidental release scenario. 


\section{Biological Agent vs. Pathogen}

Due to its unique biological and pathogenic features the outbreak strain is considered as a newly emerging pathogen (10) comparable with the serotype EHEC O157. Therefore, it can be also classified as a Category B agent (CDC). Both parameters were scored with 1 for deliberate and accidental release scenarios.

\section{Amount of the Available Agent}

A large amount of agent/pathogen that suddenly appeared and disappeared could be an indicator of a deliberate outbreak. But, there was no evidence that the outbreak strain was used as a biological agent ( 0 score for deliberate outbreak). Considering an accidental release scenario the implicated sprouts were contaminated with a significant amount of the causative pathogen taking into account the sudden increase of the number of patients and the unusual severity of diseases with increased rates of complications and deaths (1).We scored this parameter with 1 for accidental release.

\section{Means/Media of Delivery vs. Factors of Transmission}

The main mechanism of transmission during the initial explosive phase of the German Ec outbreak was alimentary and contaminated sprouts were identified as the most probable transmission factor (vehicle) of the pathogen (1). This parameter was scored with 1 for accidental release and deliberate outbreak scenarios. Obviously water, air, fomites or vectors (e.g. arthropods) were not implicated in the infection of primary cases $(0$ scores for accidental release and deliberate scenarios). In the case of a suspected deliberate outbreak identification of biological ammunition and delivery systems (e.g. aerosol generating devices) for the dissemination of biological agents in the environment would be important conclusive indicators for an intentional event. There was no evidence either for it or for aimed delivery of the pathogen through the common food chain. But, common food delivery system served for dissemination of the pathogen throughout Germany. So, we scored this parameter with 1 for accidental release and with 0 for deliberate outbreak.

A dissemination of EHEC via contaminated sprouts as a dispersion system can be considered as the most likely mechanism of release of the pathogen in the food chain. This parameter was scored with 1 for accidental release and with 0 for deliberate outbreak.

\section{Target vs. Susceptible Population at Risk}

\section{Intelligence}

With the first clusters recognized and notified 19 days after the first case, which is the maximum incubation time of EHEC O104:H4 infections, the Robert Koch Institute reacted immediately with establishing an EHEC Task Force and starting intensive epidemiological and trace-back food investigations (scored with 1 for accidental release). There were no evidences of any deliberate act (scored with 0 for deliberate outbreak).

\section{Secrecy}

German authorities did not hide any relevant information about this UEE and they promptly notified it and very openly communicated with WHO, EFSA and the European Centre for Disease Prevention and Control (ECDC) according to IHR. When the first clusters of cases were recognized by intensive national and international scientific co-operation the causative EHEC strain and contaminated sprouts as the vehicle were identified within a comparatively short time ( 0 scores for both accidental release and deliberate outbreak).

\section{Personal Control}

Personal control was implicated immediately after the outbreak was notified and contaminated vegetables were suspected to be the presumable cause and possible factor of transmission (consumer warnings, advice for food and kitchen hygiene, cease of production and distribution of sprouts, intensified control of hygiene and health of employees in food related professions, intensified recognizing of potential sources of infection). We scored this parameter with 1 for accidental release. There were no evidences that special populations have been informed or warned before UEE to avoid the suspected food ( 0 score for deliberate outbreak).

\section{Control of Means/Media of Delivery/Factors of Transmission}

Control of means/media of delivery/factors of transmission was carried out routinely according to German food law (1). Given the long incubation time of the diseases (in contrast to other EHEC infections) and delayed start of investigations caused by a late notification of the outbreak the accessibility of implicated contaminated sprouts was limited. Nevertheless, 29 of 8,062 environmental and food specimens and 7 samples of garbage, swabs and reserve specimens of meals were positive for the causative EHEC O104:H4 strain (20). Even before contaminated sprouts were suspected to be the cause of the event general consumer warnings regarding the possible infection risk of fresh vegetables had been issued. Immediately, after its identification the production and distribution of the incriminated sprouts were recalled. Therefore, we scored this parameter with 1 for accidental release. Activities of an intentional use of means/media of delivery by any actors were not observed.

There were no particular investigative, security or anti-panic measures typical for man-made disasters (deliberate outbreak scored with 0).

\section{Physical Protection Measures}

Physical protection measures regarding the populations at risk were not in place during the initial explosive phase of the outbreak and there were no chemoprophylaxis or immuno-prophylaxis against EHEC infections available. These three parameters were scored with 0 for accidental release and deliberate outbreak.

\section{Importance of Target/Population at Risk}

There were no evidences that so called "hard" targets were hit. But, the food chain is internationally considered as an important vulnerable target for criminal or terrorist acts. In industrial countries adults that are known to prefer vegetarian lifestyle and often consume meals in cafeterias or restaurants might be a "soft target”. In 2011, several agricultural companies in Germany and Spain experienced a significant financial loss due to this outbreak. However, there were no evidences or indicators that the health system or some agricultural companies in Germany and Spain were targets (score 1 for accidental release). Because Germany as a whole could have been the target we scored deliberate outbreak scenario also with 1 . 


\section{Number of People in Target/at Risk}

The parameter was not in favour of a deliberate outbreak, but of an accidental, having in mind that many consumers were occasionally exposed to contaminated sprouts (score 1 for accidental release and score 0 for deliberate outbreak).

Location and Distribution of People in Target/of People at Risk No special targets (e.g. conferences, political or cultural events) for a deliberate release could be identified. Sporadic cases and clusters of diseases were observed in all 16 German federal countries (1). Infections were associated with meals in restaurants and cafeterias but also in the households of patients. Both parameters were scored with 0 for deliberate outbreak. Considering an accidental release outbreak there was some predominance of cases in Northern and North Western federal countries of Germany (1). This was probably related to the fact that the incriminated producer in Lower Saxonia supplied its sprouts mainly to customers in these regions. Therefore, both parameters were scored with 1 for accidental release.

Final scores of the differentiation method suggest that the German Ec outbreak was most likely caused by an accidental release of a unique chimeric EHEC O104:H4 into the food chain by contaminated fenugreek sprouts. This was probably attributable to a technical accident or negligent violation of hygienic norms in the production facility (21).

\section{DISCUSSION}

From the very beginning of the German Ec epidemic, there was the vagrancy about the source of infection and mode of transmission (22). On 10 June 2011, one special charge of fenugreek seeds imported from Egypt in 2009 was announced by the German authorities in accordance with EFSA as the most probable source of contamination of sprouts, the main vehicle of the causative EHEC O104:H4 in this outbreak $(1,11,22)$.

A long lasting exposure to the pathogen, over a period of 38 days until authorities' interventions obviously became effective, indicates that an accidental release was possible in the German Ec epidemic. Despite all efforts, the incriminated strain was not isolated from the implicated seeds $(2,11)$. EFSA and ECDC strongly recommended advising consumers not to grow sprout seeds for their own consumption (24). However, it would be more probable that such an outbreak due to pure hygiene happened in Egypt rather than in Germany, but responsible Egyptian authorities declared no cases or outbreaks and denied violations of hygienic rules in the period when the exported seeds were produced (24).

It is presently accepted that seeds of different species of sprouts (e.g. raps, clover, fenugreek) could be contaminated during growth and harvesting, e.g. by natural fertilizers like animal and human faeces and urine. Another possibility is the contamination during storage by rodents, during distribution/packaging by mixing with contaminated seed lots, by false declaration of seeds, and production of sprouts for human nutrition. The persistence of EHEC on sprout seeds might be explained by the property of several gram negative bacteria species to go into a so called "viable but noncultivable state" under appropriate conditions. It is an evolutionary smart mechanism to survive, to stay transmissible and to find a new susceptible host (25).
German Ec epidemic was a point-source outbreak. King et al. (27) reported a similar outbreak in France near Bordeaux in June 2011, caused by a strain indistinguishable from German EHEC strain. They implicated contaminated sprouts of fenugreek seeds from the same source (only few lots of a shipment) as a vehicle for both outbreaks (Germany and France). Unfortunately, the causative EHEC strain was not isolated from the implicated seeds, making statements about fenugreek seeds as the source of the outbreaks questionable.

EFSA assumed that the O104:H4 clone, during the intervals between the sporadic cases, has maintained itself outside the EU. But, without solving source/reservoir of the German Ec outbreak, it is unlikely to prevent similar outbreak again in Europe. Assuming that this took place during the primary production process, this part of the investigation would need to extend beyond the point of EU import to include the site(s) of production (24).

The new hybrid EHEC strain differs in its genetic and pathogenic features from known strains of this pathogen. The new chimeric strain combining features of EHEC and EAEC is so far unique as the reservoir is human according to our present knowledge. Until spring 2011, HUSEC outbreaks had been caused only by zoonotic EHEC strains, usually $0157: \mathrm{H} 7$, and only few outbreaks were related to $\mathrm{O} 104$ strains (3). Primary transmission was by ingestion of contaminated food. However, no cases transmitted by contaminated sprouts have been reported in Germany until 2011. Direct smear infections (person-to-person) is also possible under poor hygienic conditions causing secondary and tertiary cases and a delayed course of an epidemic. But, there is no information nor evidences that EHEC 0104:H4 was deliberately "designed" or released as a biological agent.

Few strains genetically related to EHEC/EAEC O104:H4 were described already before the outbreak in Germany in 2001 (designed as HUSEC041) and 2010, in France in 2004 and 2011, in Korea and Finland in 2006 and in the Republic of Georgia in 2009(1, 28-30). The human EAEC strain from Central Africa shows 93\% similarity with EAEC O104:H4 strain (5). Interesting is E. coli $\mathrm{O} 104: \mathrm{H} 4$ strain isolated from two patients, who were a part of a cluster of diarrheal illness in the Republic of Georgia in 2009, in co-work with CDC which was similar to the German Ec strain. It produced Shiga toxin, but had a different molecular fingerprint, and was less resistant to antibiotics. There was no clear outbreak and no food identified as a vehicle of the pathogen (29). Another important is the emerging of a novel pathotype of EAEC O104:H4 especially in HIV infected people now endemic in Central Africa and spreading to Europe and Asia (20).

Compared to the median incubation period of EHEC O157 of three to four days, the median incubation period of EHEC O104 was two times longer. The average period between the onset of diarrhoea and HUS appeared to be shorter for the EHEC O104 strain than for infections with enterohaemorrhagic E. coli serotype O157 (1). The severity of the clinical course, unusually high proportion of CNS complications and higher mortality in case of hemorrhagic colitis and HUS indicate a special susceptibility of the population at risk, because no immunity against the new pathotype of EHEC could have been acquired before the outbreak. Additional contributing factor may be the initial therapy of the hemorrhagic colitis with constipating drugs (prolongation of multiplication and toxin production of EHEC) and inadequate antibiotics (increased toxin release in the gut and 
uptake of higher amounts of toxin in the circulation/distribution to kidneys and CNS).

Until the German Ec outbreak, sprouts were known as a possible but very rare vehicle in some outbreaks caused by enteric pathogens (31). In 2012 and 2014, several multistate outbreaks of gastroenteritis have been reported in the USA caused also by non EHEC O157 after consumption of contaminated sprouts in restaurants (32-34). Similar to the situation in the GHUSEC outbreak the recognition and notification of cases, microbiological identification of the difficult to cultivate strains, back tracing of food and verification of the site and mode of contamination of incriminated seeds or sprouts were reported by local, state and federal authorities to be complex and time-consuming.

Typically for food-born outbreaks German Ec had a "contact tail" with 33 cases from 5 July 2011 to the end of 2011. Some were unsolved laboratory ( 3 cases, $9.1 \%$ ) and some were nosocomial cases (15.1\%) (35). Except for usual public health recommendations and collected statistical data mainly for academic purposes (1), there were no data for better understanding of secondary cases and pathogen transmission, probably useful for new knowledge of outbreak characteristics (ways of transmission, incubation period, latency period, period of communicability), as well as improving their prevention. These data must not be obscure, because of well defined conditions and co-operability of labs and clinical staff.

EFSA indicated a high level of asymptomatic carriers and subclinical cases (24). The Robert Koch Institute reported obscure information of the longest pathogen shedding of 13 weeks without additional data (adult case included in the prospective study) (1). The conducted survey was not sensitive enough to solve the mode of transmission for some important cases (1). During the outbreak, the mean shredding period was reported to be more than 34 days for EHEC O104:H4 in patients not receiving antibiotics, with some of them still shredding the pathogen more than one year after the outbreak (2). The approximate number of asymptomatic shredders was missing. Frequency and characteristics of secondary transmission (household transmissions) have universal importance for prevention of many enteric infection diseases and not only of the German Ec.

Officially, the outbreak started on May 1st, but the public was warned for the first time on May 25th. Public warning regarding the consumption of sprouts was issued on 10 June (1). At the beginning of the outbreak, onset of disease and hospitalization dates lie well before the date of notification, which is also true for the diagnoses dates of the first cases (1). In our opinion that could be a source of "false negative" cases. In the previous 5 years (2006-2010) a median of 13 HUS cases and 218 EHEC gastroenteritis cases were reported annually. For the 2011 outbreak period, this corresponds to a 67 -fold increase in HUS and a 17-fold increase in EHEC gastroenteritis. The increase in the number of 2011 EHEC cases not ascribed to the outbreak $(n=719)$, well beyond the total EHEC cases in the previous year $(n=218)$, reflects the strongly increased attention to and higher clinical investigation rates for EHEC (1). It is also assumed that unclear/ unconfirmed cases were ascribed to current outbreak (so called "false positive cases").

The unexpectedly large size of epidemic may be connected with the modern vegetarian and vegan lifestyles with more raw food in diet. Without stringent food control this growing popula- tion may be a target for accidental (e.g. disruption of hygienic barriers) or intentional exposure to pathogens. In recent epidemics raw vegetable was an important transmission factor of enteric pathogens, which may infect or persist dormant in a viable but non-cultivable state in/on plants and their seeds (36).

The differences in the age and gender distribution of cases, especially evident in the first epidemic phase, may be explained, if sprouts are accepted as a vehicle, by higher exposure to the contaminated food. Adults consume more frequently raw vegetables than children and women are more likely vegetarians than men. Similar differences in age and gender distribution were also observed in 1994 during a foodborne outbreak of partially haemorrhagic gastroenteritis in Helena, Montana/USA caused by a rare EHEC serotype 0104:H21 producing Shiga toxin II. Most of the 18 patients were adults (median age 36 years) with a higher proportion of female cases (67\%) (37). Similar distributions of age (median 26-27 years) and gender (76-88\% females) were reported in 2012 and 2014 in two multistate outbreaks in the USA caused by Shiga toxin-producing EHEC O26 and O121 after consuming contaminated sprouts in restaurants $(33,34)$.

In conclusion, this study suggests that German Ec outbreak was a consequence of an accidental contamination of fenugreek sprouts with a new Escherichia coli strain O104:H4, probably during washing down or fertilizing on a farm in Lower Saxony. Accidents or violations of hygienic rules in production, storage, transporting and distribution of sprout seeds may cause an accidental undetectable contamination of these products. Modern industrialized large-scale food production and distribution might lead to a wide exposure of people to contaminated food like in the German Ec epidemic. Robust public health infrastructure including close disease surveillance and urgent international scientific co-operation is necessary to effectively manage and resolve foodborne outbreaks.

\section{Acknowledgement}

This study was financially supported by the Serbian Ministry of Education, Science and Technological Development, Contract No. 175078, 2010/2014.

\section{Conflict of Interests}

None declared

\section{REFERENCES}

1. Robert Koch Institute. Final presentation and evaluation of epidemiological findings in the EHEC O104:H4 outbreak, Germany 2011 [Internet]. Berlin: Robert Koch Institute; 2011 [cited 2013 Aug 20]. Available from: http://www.rki.de/EN/Home/EHEC_final_report.pdf.

2. Hauswaldt S, Nitschke M, Sayk F, Solbach W, Knobloch JK. Lessons learned from outbreaks of Shiga toxin producing Escherichia coli. Curr Infect Dis Rep. 2013 Feb;15(1):4-9.

3. Radosavljevic V, Finke EJ, Belojevic G. Escherichia coli O104:H4 outbreak in Germany - clarification of the origin of the epidemic. Eur J Public Health. 2015 Feb;25(1):125-9.

4. Rasko DA, Webster DR, Sahl JW, Bashir A, Boisen N, Scheutz F, et al. Origins of the E. coli strain causing an outbreak of hemolytic-uremic syndrome in Germany. N Engl J Med. 2011 Aug 25;365(8):709-17.

5. Mellmann A, Harmsen D, Cummings CA, Zentz EB, Leopold SR, Rico A, et al. Prospective genomic characterization of the German enterohemorrhagic Escherichia coli O104:H4 outbreak by rapid next generation sequencing technology. PLoS One. 2011;6(7):e22751. doi: 10.1371/ journal.pone.0022751. 
6. Rohde H, Qin J, Cui Y, Li D, Loman NJ, Hentschke M, et al.; E. coli O104:H4 Genome Analysis Crowd-Sourcing Consortium. Open-source genomic analysis of Shiga-toxin-producing E. coli O104:H4. N Engl J Med. 2011 Aug 25;365(8):718-24.

7. Brzuszkiewicz E, Thürmer A, Schuldes J, Leimbach A, Liesegang H, Meyer FD, et al. Genome sequence analyses of two isolates from the recent Escherichia coli outbreak in Germany reveal the emergence of a new pathotype: Entero-Aggregative-Haemorrhagic Escherichia coli (EAHEC). Arch Microbiol. 2011 Dec;193(12):883-91.

8. Bielaszewska M, Mellmann A, Zhang W, Köck R, Fruth A, Bauwens A, et al. Characterisation of the Escherichia coli strain associated with an outbreak of haemolytic uraemic syndrome in Germany, 2011: a microbiological study. Lancet Infect Dis. $2011 \mathrm{Sep}$;11(9):671-6.

9. Vonberg RP, Höhle M, Aepfelbacher M, Bange FC, Belmar Campos C, Claussen K, et al. Duration of fecal shedding of Shiga toxin-producing Escherichia coli O104:H4 in patients infected during the 2011 outbreak in Germany: a multicenter study. Clin Infect Dis. 2013 Apr;56(8):1132-40.

10. Scheutz F, Nielsen EM, Frimodt-Møller J, Boisen N, Morabito S, Tozzoli $\mathrm{R}$, et al. Characteristics of the enteroaggregative Shiga toxin/verotoxinproducing Escherichia coli O104:H4 strain causing the outbreak of haemolytic uraemic syndrome in Germany, May to June 2011. Euro Surveill. 2011 Jun 16;16(24). pii: 19889.

11. Muniesa M, Hammerl JA, Hertwig S, Appel B, Brüssow H. Shiga toxinproducing Escherichia coli O104:H4: a new challenge for microbiology. Appl Environ Microbiol. 2012 Jun;78(12):4065-73.

12. Beutin L, Martin A. Outbreak of Shiga toxin-producing Escherichia coli (STEC) O104:H4 infection in Germany causes a paradigm shift with regard to human pathogenicity of STEC strains. J Food Prot. 2012 Feb;75(2):408-18.

13. Rubino S, Cappuccinelli P, Kelvin DJ. Escherichia coli (STEC) serotype O104 outbreak causing haemolytic syndrome (HUS) in Germany and France. J Infect Dev Ctries. 2011 Jul 4;5(6):437-40.

14. Chattaway MA, Dallman T, Okeke IN, Wain J. Enteroaggregative E. coli O104 from an outbreak of HUS in Germany 2011, could it happen again? J Infect Dev Ctries. 2011 Jul 4;5(6):425-36.

15. Marejková M, Roháčová H, Reisingerová M, Petráš P. An imported case of bloody diarrhea in the Czech Republic caused by a hybrid enteroaggregative hemorrhagic Escherichia coli (EAHEC) O104:H4 strain associated with the large outbreak in Germany, May 2011. Folia Microbiol (Praha). 2012 Mar;57(2):85-9.

16. Radosavljevic V, Belojevic G. A new model of bioterrorism risk assessment. Biosecur Bioterror. 2009 Dec;7(4):443-51.

17. Radosavljevic V. A new method of differentiation between a biological attack and other epidemics. In: Hunger I, Radosavljevic V, Belojevic G, Rotz L, editors. Biopreparedness and public health. Heidelberg: Springer; 2013. p. 17-32.

18. Robert Koch Institute. Infection epidemiology yearbook [Internet]. Robert Koch Institute [cited 2016 Mar 5]. Available from: http://www.rki.de/DE/ Content/Infekt/Jahrbuch/jahrbuch_node.html. (In German.)

19. Török TJ, Tauxe RV, Wise RP, Livengood JR, Sokolow R, Mauvais S, et al. A large community outbreak of salmonellosis caused by intentional contamination of restaurant salad bars. JAMA. 1997 Aug 6;278(5):38995.

20. Rissland J, Kielstein JT, Stark K, Wichmann-Schauer H, Stümpel F, Pulz M. The EHEC O104:H4 outbreak in Germany 2011 - lessons learned?! Gesundheitswesen. 2013 Apr;75(4):184-9.

21. European Food Safety Authority (EFSA). Tracing seeds, in particular fenugreek (Trigonella foenum-graecum) seeds, in relation to the Shiga toxin-producing E. coli (STEC) O104:H4 2011 Outbreaks in Germany and France [Internet]. EFSA [cited 2014 Sep 15]. Available from: http:// www.efsa.europa.eu/en/supporting/doc/176e.pdf.
22. Responding to disease outbreaks in Europe. Lancet. 2011 Jun 11;377(9782):1978. doi: 10.1016/S0140-6736(11)60846-5.

23. Centers for Disease Control and Prevention (CDC). Outbreak of Escherichia coli O104:H4 infections associated with sprout consumption - Europe and North America, May-July 2011. MMWR Morb Mortal Wkly Rep. 2013 Dec 20;62(50):1029-31.

24. Shiga toxin-producing E. coli (STEC) O104:H4 2011 outbreaks in Europe: Taking Stock. EFSA Journal 2011;9(10):2390. doi:10.2903/j. efsa.2011.2390.

25. Aurass P, Prager R, Flieger A. EHEC/EAEC O104:H4 strain linked with the 2011 German outbreak of haemolytic uremic syndrome enters into the viable but non-culturable state in response to various stresses and resuscitates upon stress relief. Environ Microbiol. 2011 Dec;13(12):3139-48.

26. Buchholz U, Bernard H, Werber D, Böhmer MM, Remschmidt C, Wilking $\mathrm{H}$, et al. German outbreak of Escherichia coli O104:H4 associated with sprouts. N Engl J Med. 2011 Nov 10;365(19):1763-70.

27. King LA, Nogareda F, Weill FX, Mariani-Kurkdjian P, Loukiadis E, Gault $\mathrm{G}$, et al. Outbreak of Shiga toxin-producing Escherichia coli O104:H4 associated with organic fenugreek sprouts, France, June 2011. Clin Infect Dis. 2012 Jun;54(11):1588-94.

28. Prager R, Lang C, Aurass P, Fruth A, Tietze E, Flieger A. Two nove EHEC/EAEC hybrid strains isolated from human infections. PLoS One. 2014 Apr 21;9(4):e95379. doi: 10.1371/journal.pone.0095379.

29. Centers for Disease Control and Prevention. Reports of selected E. coli outbreak investigations [Internet]. Atlanta: CDC [cited 2012 Apr 12]. Available from: http://www.cdc.gov/ecoli/outbreaks.html.

30. Wu CJ, Hsueh PR, Ko WC. A new health threat in Europe: Shiga toxinproducing Escherichia coli O104:H4 infections. J Microbiol Immunol Infect. 2011 Oct;44(5):390-3.

31. Michino H, Araki K, Minami S, Takaya S, Sakai N, Miyazaki M, et al Massive outbreak of Escherichia coli O157:H7 infection in schoolchildren in Sakai City, Japan, associated with consumption of white radish sprouts. Am J Epidemiol. 1999 Oct 15;150(8):787-96

32. Ergebnisse des workshops: Experiences from the STEC O104:H4 outbreak in Germany and research needs for STEC". Epidemiologisches Bulletin. 2012;9(5):71-4. (In German.)

33. Centers for Disease Control and Prevention. E.coli (Escherichia coli) Multistate outbreak of Shiga toxin-producing Escherichia coliO26 infections linked to raw clover sprouts at Jimmy John's restaurants (final update) [Internet]. Atlanta: CDC; 2012 [cited 2014 Sep 9]. Available from: http://www.cdc.gov/ecoli/2012/O26-02-12/index.html.

34. Centers for Disease Control and Prevention. E.coli (Escherichia coli). Multistate outbreak of Shiga toxin-producing Escherichia coliO121 infections linked to raw clover sprouts (final update) [Internet]. Atlanta: CDC; 2014 [cited 2014 Sep 9]. Available from: http://www.cdc.gov/ ecoli/2014/O121-05-14/index.html.

35. Frank C, Milde-Busch A, Werber D. Results of surveillance for infections with Shiga toxin-producing Escherichia coli (STEC) of serotype O104:H4 after the large outbreak in Germany, July to December 2011. Euro Surveill. 2014 Apr 10;19(14). pii: 20760.

36. Schikora A, Carreri A, Charpentier E, Hirt H. The dark side of the salad: Salmonella typhimurium overcomes the innate immune response of Arabidopsis thaliana and shows an endopathogenic lifestyle. PLoS One. 2008 May 28;3(5):e2279. doi: 10.1371/journal.pone.0002279.

37. Centers for Disease Control and Prevention (CDC). Outbreak of acute gastroenteritis attributable to Escherichia coli serotype O104:H21-Helena, Montana, 1994. MMWR Morb Mortal Wkly Rep. 1995 Jul 14;44(27):5013 . 